\title{
Energy Analysis of Services through Green Metrics: Towards Green Certificates
}

\author{
Mariagrazia Fugini $^{1}$ and José Antonio Parejo Maestre ${ }^{2}$ \\ ${ }^{1}$ Dipartimento di Elettronica e Informazione \\ Politecnico di Milano \\ Piazza L.daVinci, 32, I-20133 Milano, Italy \\ fugini@elet.polimi.it \\ ${ }^{2}$ Dpto. de Lenguajes y Sistemas Informáticos \\ E.T.S. de Ingeniería Informática \\ Avda. Reina Mercedes, s/n.41012 Sevilla, Spain \\ japarejo@us.es
}

\begin{abstract}
Energy-awareness in services can be obtained through annotations regarding energy consumption. In this paper, annotations are given as Green Performance Indicators (GPIs). A service is annotated in terms of its structure, of the used IT platform, and of development costs, human resources, and environment impact. GPIs relate to service development, deployment, and maintenance. An approach is proposed based on monitoring the GPIs so as to enable the analysis of services from their energy consumption viewpoint. Our approach allows estimating energy efficiency of services through a comparison of behaviorally similar services (e.g., an on-line purchasing service) through the analysis of their GPIs. By collecting details from GPIs, we propose a model for an energy certificate, called Green Certificate, aimed at classifying services at given energy efficiency levels according to the energy they consume during their lifecycle.
\end{abstract}

Keywords: Energy consumption, Green computing, Service Systems, Performance Indicators, Metrics, Service Energy Accounting, Green Certificate.

\section{Introduction}

Under a green IT approach, services can be described in terms of the energy they consume. Energy depends of software/hardware resource employed by the service to run and of the resources they require along their lifecycle, even from an organizational viewpoint. Energy-related issue are gaining attention in academia and industry by developing services that can be tuned to consume less energy in terms of power, IT resources, employed human resources or consumable/supply chains, and so forth [2, 13].

To sustain this attention, it is necessary to stress the importance of designing, developing, and executing service-based applications along the perspectives of energy awareness $[3,8]$. This means that services can be characterized by metrics regarding which resources and of what type (e.g., processor, memory, but also consumables or human resources) required by the service to run, as well as how much effort (e.g., in terms of costs, to consider a unique standard metric) it requires during development, deployment, execution, and maintenance along its lifecycle. 
In this paper, we characterize services in terms of properties featuring their energy consumption in data centres. These properties are specified as energy-awareness parameters or annotations called Green Performance Indicators (GPIs). We show how a service can be annotated with GPIs describing the service in terms of the IT resources it uses (CPU, memory, storage, and so on), of the organizational factors, e.g., human resources, involved in its development and management, and in terms of its impact on the environment, such as consumables directly or indirectly produced within the service lifecycle. We also refer to energy consumption vs Quality of Service (QoS) as an indicator of to which extent a consumer is willing to accept, e.g., lower response time in front of energy saving.

The paper shows that, through a GPI annotation, the service can receive an energy certificate, called Green Certificate (GC), which is a tradable commodity for service providers and consumers. The GC shows the energy consumption level of a service from various viewpoints: from the technical side (IT or provider's perspective) and from the organizational side (business or customer's perspective). The ultimate purpose of the GC is to support service selection form service registries taking into account also energy criteria, besides service behavior and service non-functional issues (e.g., response time, availability, QoS, security level).

We rely on our previous work on GPIs for the definition of a comprehensive set of GPIs providing a global view of energy consumption along the service provisioning model, from service strategy, design and development, to execution, control and monitoring. In fact, this research is carried out in the EU-FP7 GAMES Project ${ }^{1}$. GAMES develops methodologies, software tools, and innovative metrics for an energy-aware design and management of service centers [2]. It proposes guidelines for designing and managing service-based applications along the perspectives of energy awareness. The approach focuses on: a) co-design of energy-aware applications and their underlying services and IT architectures in order to satisfy users, context, and Quality of Service (QoS) requirements, addressing energy efficiency and controlling emissions. This is carried out through the definition of GPIs to evaluate if and to what extent a given service and workload configuration will affect the carbon footprint emission levels; b) run-time management of IT service centre energy efficiency, exploiting an adaptive system behavior at run time. GAMES relies on web services as a suitable support to adaptivity to different system states and requirements in front of energy policies. GAMES defines a green lifecycle for development of adaptive, self-healing, and self-managing application systems able to reduce energy consumption. In particular, in GAMES, we have defined GPIs and have shown the results of monitoring complex service applications using a monitoring technique based on the analysis of GPIs at the IT infrastructure level. This paper makes a step forward in the definition of a Green Certificate out of GPIs. To this aim we consider a subset of all the defined GPIs and show their WS-* compliant specification to be stored in a registry of service descriptions.

The paper is organized as follows. Section 2 reviews related work. Section 3 describes GPIs and how we analyze energy efficiency for a given service. Section 4 introduces the fundamentals of a GC specification and use. Section 5 concludes the paper. 


\section{Related Work}

Research on metrics for green IT and data centres are in progress with the vision of achieving economic, environmental, and technological sustainability. Several sets of metrics are available to measure data centre efficiency, proposed by Green $\mathrm{Grid}^{2}$, Uptime Institute $^{3}$, Transaction Performance processing Council (TPC) ${ }^{4}$ and others. However, there is no widely accepted metric set [12], allowing for easy measuring and monitoring of energy consumed and wasted by a data center. In [10] a set of server energy efficiency measures and metrics is presented. It envisions the requirements for new metrics considering Green IT as a technology to be harmonized as a hardware, software, architecture, and QoS solution. The research towards energy efficient software began with estimating the energy consumption of the processor by instruction level power models [14]. Earlier research studies on energy efficiency of software addressed methodologies that target memory related power consumption [15]. However, in all these research works, the energy consumption of a given application is estimated upon the given hardware architecture. In [8], we have presented a new energy efficiency metric for a single service, which maps directly the relationship between energy consumption and execution time. In this way, we can compute both quality and energy metrics for each service and design a novel constraint-based quality and energy-aware service composition. In our current approach, we have considered green metrics for service applications from the hardware usage, the service lifecycle, as well as for the environmental and organizational factors perspectives. Energy efficiency is defined as the ratio between the energy used to reach the goal and the total energy consumed. For instance, one of the most used metric to measure electrical efficiency in Data Centers in the Data Center infrastructure Efficiency (DCiE), calculated by dividing IT equipment power by total facility power. DCIE was developed by members of the Green $\mathrm{Grid}^{5}$, an industry group focused on data center energy efficiency. Here, we consider energy consumption by a service as a factor that depends on processor usage, data storage usage, and I/O peripheral usage, as well as from organizational factors. We say that a service performs in a "green way" if it delivers the expected results according to given Quality of Service (QoS) requirements consuming less processor and/or less storage and/or less I/O or less organizational elements. Our approach is ultimately an adaptation of the Activity Based Costing (ABC) approach [3], tailored to the specific characteristics of services based activities and applied to the evaluation of the energy consumption through costs. $\mathrm{ABC}$ identifies activities in an organization, and assigns the cost of each activity with resources related to all services, according to the actual consumption by each. ABC has been criticized because of the high costs of modeling and inefficiency when manually driven, which had historically leaded to the adoption of alternative approaches [5]. However, current IT services infrastructure

2 www.thegreengrid.org

3 www.uptimeinstitute.org

4 www.tpc.org 
capabilities for monitoring and automated data capturing make it affordable and viable. The convenience of $\mathrm{ABC}$ for the analysis of service-based economic activities has been pointed-out in literature [5] due to its holistic view of the process of service provision, taking into account the total costs.

\section{Green Performance Indicators (GPIs)}

GPIs as design-time annotations of a service are aimed at providing information that allows designers to provide a better design for activities (e.g., lowering the amount of used data or of human resources needed to design and to fix the code/tune the deployment). The approach in this paper aims at obtaining a tool supporting the analysis of energy efficiency through analysis of GPIs. GPIs can be examined to identify energy peaks and their reasons by comparing similar services (namely, services having the same functional behavior with possibly different non-functional properties, e.g., with higher response time or lower availability). The purpose is to discover whether, by using a different service, for instance a less processing-intensive service, the same activity can be executed with the same functional results, under a customer-accepted response time, and with less energy consumption.

GPIs are structured in four clusters:

- IT resource usage metrics, related to the use of the IT platform;

- Lifecycle metrics, describing the process lifecycle expenditures (costs of modelling, analysis, design, development, deployment, maintenance, and evolution) and QoS metrics.

- Energy impact metrics, related to the impact of the application lifecycle on the environment (considering electricity, power supply, consumed material, and emissions).

- Organizational metrics, taking into account human factors involved in running and managing applications, and the standardizations and compliance efforts to obtain more energy-efficient systems.

As detailed in [7], the GPIs are layered according to a classical pyramid of information and business systems [9], namely at the strategic, tactic/control, and operational level, as reported in Fig. 1. At the strategic level [4], we insert GPIs able to drive high-level decisions about a system organization in terms of used human resources, impact on the environment, outsourcing of non-core services, guidelines for system development according to eco-related laws and regulations (such as policies of Energy Star ${ }^{6}$, United Nations Global Compact ${ }^{7}$ etc.), and so on.

6 ENERGY STAR http://www.energystar.gov/

7 United Nations Global Compact http://www.unglobalcompact.org/ 
GPIs at the tactic/control level denote how the service will consume less energy if its development is enhanced, e.g., through the use of mature platforms, which will improve the system quality in terms of service delivery versus customers' expectations and in terms of less complexity of the service interfaces. Decommissioning of unused services and data is a GPI at this level, controlling the recovering of system resources. GPIs about consumables measure to what extent the system is wasting in terms of paper, toners, materials, and so on. At the operational level, we insert GPIs for monitoring the energy related to usage of IT resources, such as the processor, the $\mathrm{I} / \mathrm{O}$, and the storage. We consider these factors as a characterization of energy needs that keep GPIs independent of physical configurations of machines and storage devices.

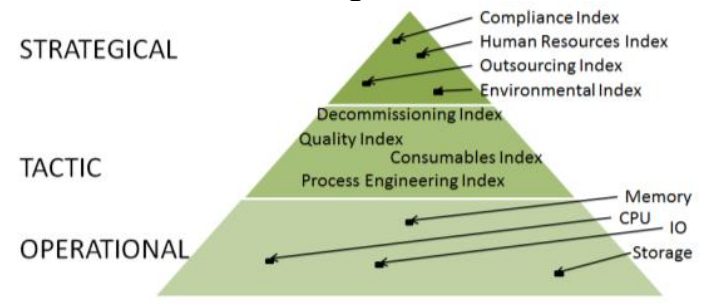

Fig. 1. Layered GPIs

\subsection{Principles to derive a Green Certificate}

In order to set the principles for obtaining a $\mathrm{GC}$, in this paper, we make the following hypothesis. First we will consider a subset of the GPIs defined in GAMES [8] in order to simplify the formulation of the GC and the computation of the costs. We have selected a set of GPIs which are listed in Table 1. The selection has been performed on a pure sampling basis, avoiding the most complex GPIs. For Units, we consider costs units as a uniform measurement basis. Secondly, GCs regard services, not complex business processes, whole applications or products. The motivation is that we aim at having a clear boundary for factors to be measured to compute the values of the GPIs. Third, the proposed method for creation and use of the GC is intended for comparisons and selection of services. In particular, we consider functionally equivalent services and provide criteria for analyzing energy consumption in a comparative way.

\subsection{Comparison-based approach for energy analysis of services}

The approach compares similar services considering their energy-related annotations. GPIs are considered for pairs of services (or service groups). We separate the technical annotations (IT view) of a service from its organizational annotation (business view), in order to take into account the total energy consumption related with service provisioning. As stated, for the sake of simplicity, in what follows we express energetic consumption as costs. This allows us to aggregate measurements related to different activities, and provides a single significant value for groups of related GPIs (named henceforth GPI Clusters). The importance of the annotation of services for energy analysis has been expressed in our previous work [5, 3]. 
Table 1. A subset of GPIs selected for illustration of GC

\begin{tabular}{|c|c|c|}
\hline GPI Cluster & GPI & Definition (expressed as costs) \\
\hline \multirow[b]{2}{*}{ Strategic } & $\begin{array}{l}\text { Human Resources } \\
\text { Index (HR) }\end{array}$ & $\begin{array}{l}\text { Cost of human involvement in service development, } \\
\text { deployment execution and management. }\end{array}$ \\
\hline & Environmental Index (E) & $\begin{array}{l}\text { Costs associated to } \mathrm{CO} 2 \text { emissions, water, energy } \\
\text { consumption, etc. caused by production, } \\
\text { transportation, logistics, etc. involved in service } \\
\text { lifecycle. }\end{array}$ \\
\hline \multirow{2}{*}{ Tactic/Control } & Decommissioning Index (D) & $\begin{array}{l}\text { Cost associated to the energy that can be re-generated } \\
\text { by non-usage, versioning, or substitution of services. }\end{array}$ \\
\hline & Consumables Index (C) & $\begin{array}{l}\text { Costs associated to printouts and material produced } \\
\text { by the service during execution. }\end{array}$ \\
\hline \multirow{4}{*}{ Operational } & CPU (cpu) & Cost of power consumption by CPU \\
\hline & $\mathrm{IO}(\mathrm{IO})$ & Cost of power consumption by I/O operations \\
\hline & Memory Usage (M) & Cost of power consumption by Memory \\
\hline & Storage (S) & $\begin{array}{l}\text { Cost associated to the energy consumption of the long } \\
\text { term storage subsystem (can be independent of the } \\
\text { main } \mathrm{I} / \mathrm{O} \text { and even be replicated). }\end{array}$ \\
\hline
\end{tabular}

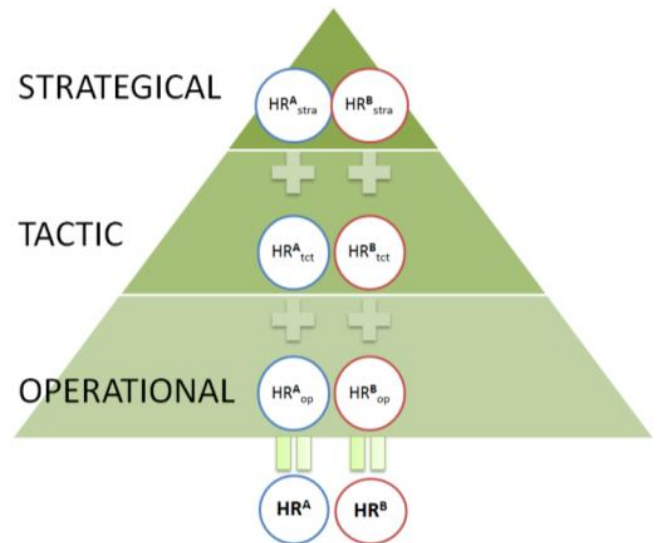

Fig. 2 Splitting the values of the Human Resources GPI into its level components: comparing two services A and B

To compute the values of the GPIs, we consider that a service is exposed through its WSDL (Web Service Definition Language) enriched with GPIs at the three levels, as an in information systems for service management. Subsequently, we relate GPIs at one level with the technical metrics that contribute to obtain that GPI. By considering the same three levels also for the technical metrics of the services, we support the analysis of an organizational GPI into its technical metrics.

An example is the Human Resources (HR) GPI, related to monitoring how much programmers, or service maintenance personnel "consume" in terms of man/hour to keep services on line and available (this is a tactic/control-level GPI). This example is depicted in Fig. 2. By checking this GPI, an organization manager can explore the technical causes determining the energetic cost related to HR. He can than decide that endowing a 
programmer with an automatic maintenance tool can help to save energy since the maintenance is less expensive and hence the service is more energy performing. More precisely, the HR GPI considered from an organizational viewpoint can be analyzed in its three-layer technical components (operational, tactic/control, strategic), going in depth about the factor(s) which most heavily contribute to energy consumption. This analysis can be performed by automated tools (which we are outlined in the following of the paper) by bringing a global HR down to its elementary components. These components can be simply summated as follows to create the global HR value:

$$
\mathrm{HR} \text { (service_A) }=\mathrm{HR}_{\mathrm{op}}+\mathrm{HR}_{\text {tct }}+\mathrm{HR}_{\text {strat }}
$$

Other GPIs are not so simple to be split, since their global value cannot be obtained as a summation but is sometimes a means or even requires weighted combinations.

An issue to be taken into account in the computation of GPIs values is that some of the costs must be distributed and balanced over a set of services (e.g., using weights). Moreover, the cost computation should be linked to a time dimension namely be timeframed observation interval (monthly, annually, etc.), and consequently the cost accounting should focus on the appropriate period. For instance, the total cost associated to the consumables index could be computed on a per-year basis, by dividing the total consumables cost of the infrastructure supporting the services of an organization, and dividing it by the number of services hosted, and the number of invocations (along this year) to which the certificate refers. As a final remark, we note that the time-frame should be consistent among all the GPIs taken into account in a GC if global and per-GPIcluster costs are provided.

Providing a full formulation for computing the values for each one of the selected GPIs is out of the scope of this paper, but will be addressed in future work. Of course, the huge volume of data to deal with and the wide range of aspects included in GPIs can be a source of uncertainty, since most of the data that has to be included is hard to measure. Consequently, the propagation of the uncertainty related to those measurements should be done in order to demonstrate that an index of abstract level with this wide range and data is able to really measure something in real life problems. Two examples concerning this problem are as follows. 1) The human Resources Index, for instance, is still hunted by the problems described in [6]. Beyond these old problems, the question arises e.g. how to distribute the energy wastes between small teams working at home with low travel cost or employees working miles away or distributed teams using a lot of communication technology etc. 2) What if a strategy implies a waste of resources on the operation level in one project context, and in the other contexts this effect is quite small and so the advantages of this particular strategy lead to a low use of energy? As 2) shows, our GPIs should also deal with the question of unique measurability and with the problem of a kind of "Bullwhip-Effect" in the composed index values which might occur by escalating uncertainties. As 1) indicates, it should be studied further how GPIs are assumed to be measured as abstract index values without too wide uncertainties. These aspects will be addressed when dealing with composed services, which are currently out of the scope of this paper. Namely, it may be not simple to apply the additive property given here, and hence a discussion about the questions above can extend the approach to energy measures presented in this paper. 


\section{Specifying and Using Green Certificates}

The Green Certificate (GC) is proposed as a document that qualifies a service at a given level of "greenness". It has three main sections: Issuer Declaration, GPI Catalog and Valuation. Fig. 3 shows the UML meta-model of its structure. The IssuerDeclaration section is a placeholder where the issuer of the certificate can insert his name, the address of his web site or contact mail, and a signature for the certificate such that the integrity and authenticity of the valuations section could be guaranteed. To this purpose, we have integrated the GC definition with the XML Data Signature W3C standard http://www.w3.org/TR/xmldsig-core/). This paves the way for the specification certificates whose issuers can be different organizations, such as the service developers and owners of the platform where the service is deployed. These organizations could play the role of a trusted third party in charge of monitoring and auditing the values of the GPIs.

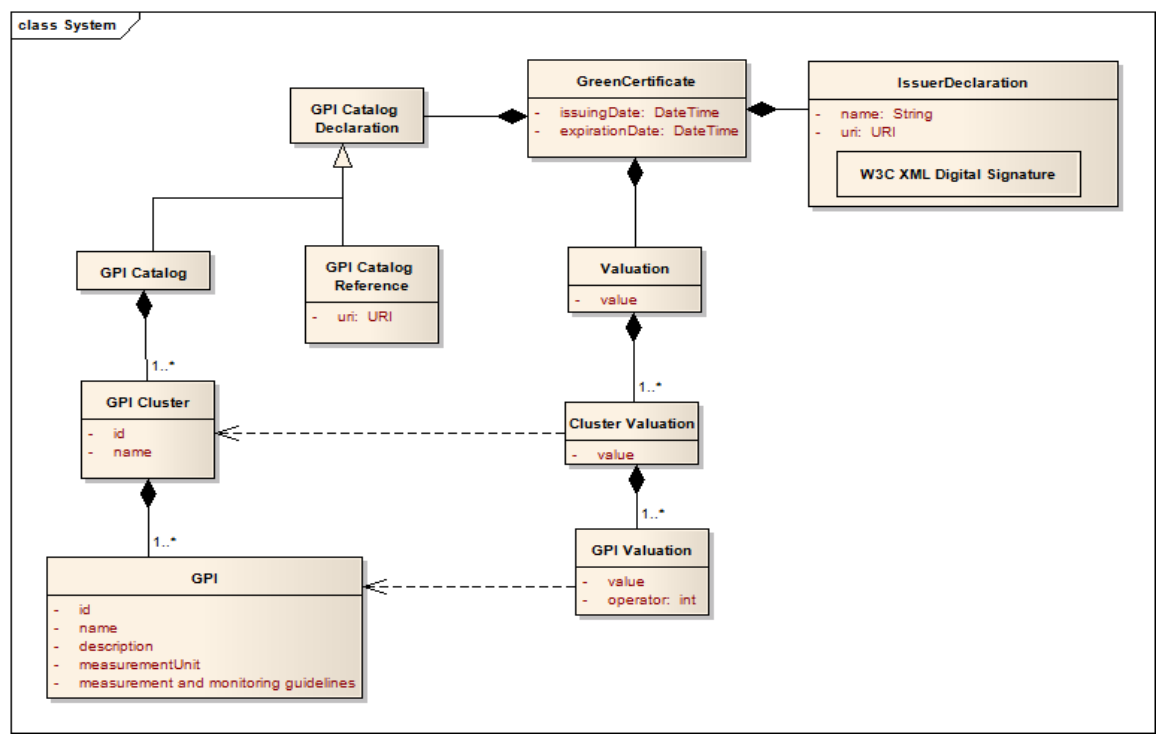

Fig.3 UML meta-model of Green Certificates

The GPI Catalog section contains the definitions of GPI Clusters and single GPIs in the clusters that will have an assigned value in the GC. We left open the possibility of specifying the Catalog of GPIs by creating a global reference through an URL. This allows for the separation between the definition of the catalog of GPIs and the definition of the GC. This also allows for the reuse of GPI Catalogs. In this sense, catalogs can be stored in separate documents and referenced from multiple certificates. To demonstrate this feature, we have created a GPI catalog named "The GAMES Canonical GPI Catalog", where our chosen set of GPIs is defined. New GCs can use this basic GPI catalog as a starting point. For each GPI, the catalog can define: an identifier, a name, a unit of measurement, a description, and monitoring and measurement guidelines written 
in natural language. The Valuation section of the GC specifies the costs for the GPI, its aggregation by clusters and even the global cost value are provided.

\subsection{Use of the Green Certificate: Provider and Consumer perspectives}

Providers have access to all the details of GPIs for cost computation and can perform analysis on them based on the hierarchical diagrams of GPIs shown in Fig.2. We also offer the possibility of using Kiviat diagrams, which display multivariate data in a twodimensional chart of three or more quantitative variables represented on axes starting from the same point. Authors consider that this kind of chart are especially useful, since they allow to simultaneously evaluate the global cost as the area of the polygon shown in the chart and the specific costs of concrete GPIs in its corresponding axis. Moreover, when GPI clusters are present, authors recommend the use of an additional separator axis between them, which allows the graphical displaying of the global cost of each GPI Cluster as an independent polygon. In Fig. 4 these two kinds of Kiviat diagrams are shown. In this case, they are used for comparing the GPIs of two different services.

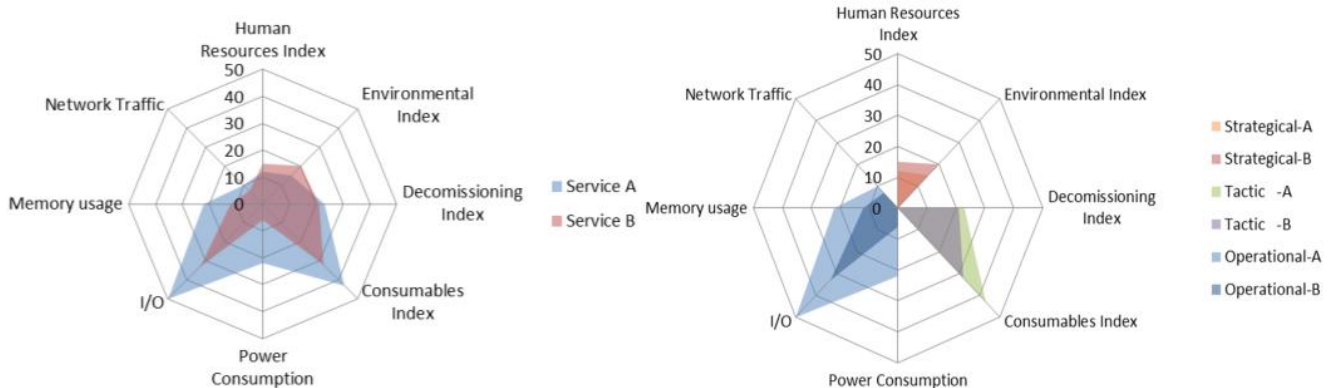

Fig. 4 Two Kiviat diagrams. The left-side diagram shows the whole cost of services, the rightside one uses additional separator axes in order to show global costs per GPI Cluster.

Service Providers expose a certificate with Green values for clusters and, on demand, for single GPIs. Service Consumer can use the Certificate in different ways, namely:

1. Select the service that has a "better" Certificate in terms of global cost.

2. Select a service according to an analysis of costs of the clusters, e.g., select service that has the lowest cost for IT cluster.

3. Select a service according to an analysis of cost of the single GPI, e.g., select service that has lowest cost in storage occupancy.

Regarding these three selection criteria, we must notice that a cost is always related to an acceptance range from the customer (not related to an absolute point or exact value) because we cannot create an absolute category of service costs for all kinds of application domains. Moreover, even for a given category of services, e.g., scientific computation services, costs cannot be evaluated for the single service in an absolute way but rather only in a comparative way, by comparing two (or more) services having the same functionality.

In order to select the most suitable service for the specific customer, some more detailed analysis of the causes of a cost can be performed at the cluster level or even at 
the single GPI level. The level of analysis the customer can perform depends on the provider/customer relationship and on market considerations. For example, the level can depend on the consumer's goals in terms of cost reduction. So, for instance a provider can expose a Green Certificate analyzable at the global level only if he wants to provide the service as a black box executed at his site on his IT infrastructure. Conversely, if he allows a service to be deployed on the customer's site, he should expose the Green Certificate with details at the strategic and tactic levels only, while providing no details of the GPIs regarding the operational level.

Finally, some customers might want to know the details of the area where they are expending for energy: so the detail of analysis level will be eventually decided on a market convenience basis. A contract negotiation will be the basis for admitted/available levels of analysis.

In order to support and aid the creation of green certificates, an XML schema has been created. This schema specifies the structure and acceptable values of the elements of GCs and can be used by editors to validate them. Moreover, we have created two XSLT transformations for the green certificates. XSLT transformations allow the automated generation of HTML or PDF documents from XML. On the one hand, we have created a "document XLST", that transforms a GC into a human readable document with a set of tables where the values of the GPIs are shown. On the other hand, we have created a "Graphical Representation XSLT" that shows the Kiviat diagram of this certificate using a set of APIs and functions for image generation provided online freely by Google as a service. A sample GC along with the corresponding results generated by the transformations can be found http://www.isa.us.es/uploads/GAMES/TranformationDemo.html.

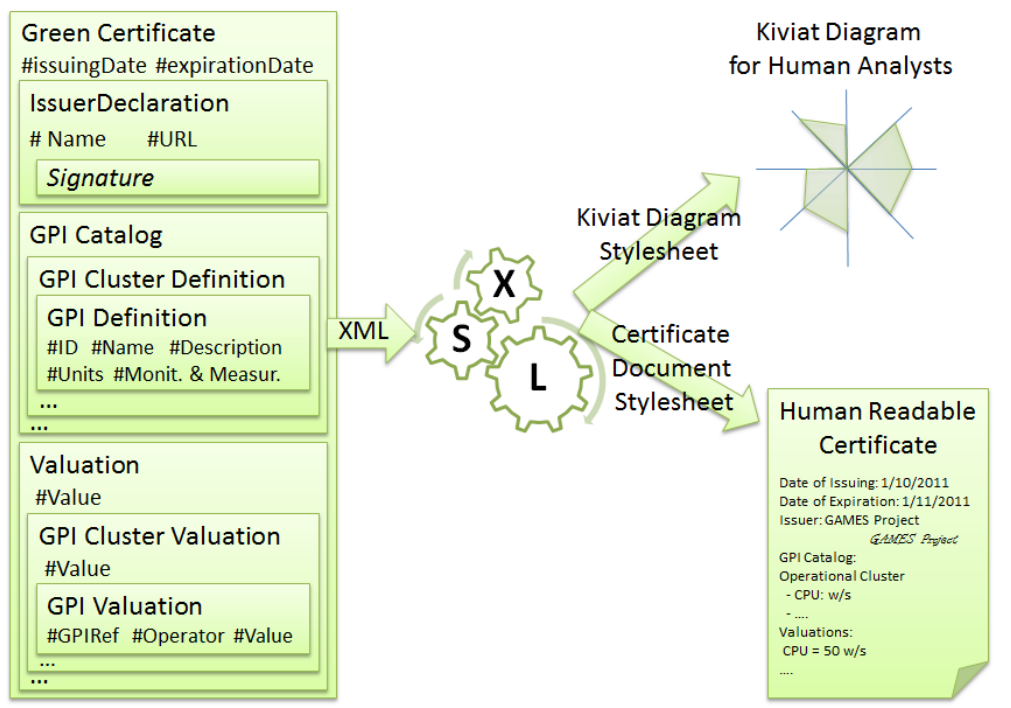

Fig. 5 Structure of GCs and transformations through XSLT to different document formats 


\subsection{Integration of the Green Certificate with the WS-* standards stack}

A set of standards have been promoted by different organizations (such as the W3C, or OASIS) for the specification of functional and non-functional properties of services. On the functional side, the main standard is WSDL, that allows describing the set of operations, and input/output data formats of services. On the non-functional side, there are various proposals usually called the WS-* standards stack, but we will focus on WSPolicy and WS-Agreement. On the one hand, WS-Policy [11] is a W3C recommendation that provides a framework for expressing domain-specific capabilities, requirements, and general characteristics of web services, named policies. Those policies can be inserted in the WSDL contract of the service allowing providers to advertise specific non-functional properties of their services. From the client perspective, those policies allow for a more precise and enriched discovery and selection of services based on such non-functional properties. On the other hand, WS-Agreement [1] is a specification by the Object Grid Forum that aims at enabling the creation of offers and electronic contracts between service providers and customers. WS-Agreement documents contain a set of guarantee terms, which specify guarantees on the values of the non-functional properties of the services affected by the agreement. The specific XML language in which guarantee terms are expressed is left open in the specification, thus GCs could be used to specify the energy consumption costs associated to the service as a guarantee of the contract or offer. The use of XML as the underlying format for GCs and the specification of an XML schema for describing their structure enable a seamless integration of our proposal with the WS-* standards stack we have created an the creation of Green Certificates.

As a demonstration of the applicability of the proposal and its compatibility with WSAgreement and WS-Policy, a sample WS-Agreement contract and WSDL interface definition enriched with GCs for specifying non-functional properties of services are available at http://www.isa.us.es/uploads/GAMES/Agreement-GC.xml and http://www.isa.us.es/uploads/GAMES/WSDL-GC.xml respectively. Those examples demonstrate that the framework proposed in this paper can be applied to service selection scenarios. However the integration of the GC into current selection algorithms and tools and its application on simulations scenarios is proposed as future work.

\section{Conclusions}

The paper has described a method for energy consumption analysis, and developed the concept of Green Certificate. GPIs have been presented which enable evaluating energy consumption in services starting from the analysis of the overall resources used/required by the service. The organizational factors related to a service lifecycle have also been considered, analyzing in particular the case in which services selection from the providers/customers can be augmented by using analysis tools to evaluate the GC exposed for the service as an energy certificate. The concept GPIs and GC for energyawareness can be pursued in several directions. First, the GC schema can be related to service in a more complex yet significant way by providing formulas to split costs of GPIs into elementary costs. In GAMES, further work will also consider tagging the services provided by data centers with their technical and business characteristics, form 
the IT infrastructure and facilities, to strategic and control issues that contribute to the energy consumption associated to a service.

Acknowledgments. This work has been partially supported by the European Commission (FEDER) project SETI (TIN2009-07366), by the project ISABEL (P07-TIC-2533) funded by the Andalusian local Government, and by the GAMES project (http://www.greendatacenters.eu/), partly funded by the European Commission's IST activity of the 7th Framework Program (contract ICT-248514). This work expresses the opinions of the authors and not necessarily those of the European Commission.

\section{References}

1. Alain Andrieux, Karl Czajkowski, Asit Dan, Kate Keahey, Heiko Ludwig, Toshiyuki Nakata, Jim Pruyne, John Rofrano, Steve Tuecke, and Ming Xu. Web services agreement specification (wsagreement). Specification from the Open Grid Forum (OGF), 03, 2007.

2. Massimo Bertoncini, Barbara Pernici, Ioan Salomie, and Stefan Wesner. Games: Green active management of energy in it service centres. In Pnina Soffer, Erik Proper, Wil Aalst, John Mylopoulos, Michael Rosemann, Michael J. Shaw, and Clemens Szyperski, editors, Information Systems Evolution, volume 72 of Lecture Notes in Business Information Processing, pages 238252. Springer Berlin Heidelberg, 2011.

3. Cinzia Cappiello, Mariagrazia Fugini, G. R. Gangadharan, Alexandre Mello Ferreira, Barbara Pernici, and Pierluigi Plebani. First-step toward energy-aware adaptive business processes. In OTM Workshops, pages 6-7, 2010.

4. Fred R. David, How companies define their mission, Long Range Planning, Volume 22, Issue 1, pages 90-97, February 1989.

5. Peter F. Drucker. Management Challenges in the 21st Century. HarperBusiness, 1st edition, 1999.

6. Frederick P. Brooks. The Mythical Man-Month: Essays on Software Engineering. AddisonWesley. Anniversary Edition. 1995

7. Mariagrazia Fugini, G.R. Gangadharan, and Barbara Pernici. Designing and managing sustainable IT service systems. in Competitive and Sustainable Manufacturing, Product and Services (APMS) Conference, Cernobbio, Italy, Oct. 11-13, 2010.

8. Alexander Kipp, Tao Jiang, and Mariagrazia Fugini. Green metrics for energy-aware IT systems CISIS, pages 241-248, 2011.

9. James A. O'Brien and G. M. Marakas. Management Information Systems. McGraw-Hill, 2011.

10. Sekiguchi, S. Itoh, M. Sato, and H. Nakamura. Service aware metric for energy eficiency in green data centers. http://www.iea.org/work/2009/standards/Sekiguchi.pdf, 2009.

11. Asir S Vedamuthu, David Orchard, Frederick Hirsch, Maryann Hondo, Prasad Yendluri, Toufic Boubez, and Ümit Yalçinalp. Web services policy 1.5 framework. W3C Recommendation, September 2007.

12. Joseph Williams and Lewis. Curtis. Green: The new computing coat of arms? IT Professional, 1(10):12-16, 2007

13. Stavros Harizopoulos, Mehul Shah, and Parthasarathy Ranganathan. Energy efficiency: The new holy grail of data management systems research. In: Proc. 4th Biennial Conference on Innovative Data Systems Research (CIDR). 2009.

14. Vivek Tiwari, Sharad Malik, and Andrew Wolfe. Power analysis of embedded software: A first step towards software power minimization. IEEE Trans. on VLSI Systems, 2:437-445, 1994.

15. Alexandre M. Ferreira, Kyriakos Kritikos, and Barbara Pernici. Energy-aware design of servicebased applications. In L. Baresi, C.-H. Chi, and J. Suzuki, editors, ICSOC/ServiceWave, volume 5900 of Lecture Notes in Computer Science, pages 99-114, 2009. 